PROCEEDINGS OF THE

AMERICAN MATHEMATICAL SOCIETY

Volume 125, Number 7, July 1997, Pages 1885-1891

S 0002-9939(97)04106-3

\title{
ON THE VIRTUAL COHOMOLOGICAL DIMENSIONS OF COXETER GROUPS
}

\author{
A. N. DRANISHNIKOV
}

(Communicated by James West)

\begin{abstract}
We apply Bestvina's approach of calculation of the virtual cohomological dimension of Coxeter groups. The explicit formula for $v c d_{F} \Gamma$ in terms of cohomological properties of the corresponding complex $K$ is given.
\end{abstract}

\section{INTRODUCTION}

If an Eilenberg-Mac Lane complex $K(\Gamma, 1)$ of a group $\Gamma$ is finite, then the cohomological dimension $c d_{F} \Gamma$ of the group $\Gamma$ with respect to an abelian group $F$ can be defined as a maximal number $n$ such that $H_{c}^{n}(\bar{X} ; F) \neq 0$, where $\bar{X}$ is the universal cover of $K(\Gamma, 1)$. According to the theorem of Serre [9] if $c_{F} \Gamma<\infty$ for a finite index subgroup $\Gamma$ of the group $G$, then the number $c d_{F} \Gamma$ does not depend on a choice of $\Gamma$. That number is called the virtual cohomological dimension of $G$ with respect to $F$ and is denoted by $v c d_{F} \Gamma$.

For every Coxeter group $\Gamma$ Bestvina has constructed a finite polyhedron $B(\Gamma, F)$ such that $v c d_{F} \Gamma=\operatorname{dim} B(\Gamma, F)$ for sufficiently good $F[1]$. Using his result we give a formula for $v c d_{F} \Gamma$ for Coxeter groups in terms of cohomologies of Davis' panel complex. Then we apply that formula to studying the topology of the boundaries of Coxeter groups. First we get a new proof of the fact that every Pontryagin surface can be a boundary of a Coxeter group [2] (see also [1], Remark 3). Second, the formula implies that the Boltyanski compactum cannot be a boundary. Thus, the logarithmic law can fail for virtual dimension of the product of two Coxeter groups, i.e. there are groups $\Gamma_{1}$ and $\Gamma_{2}$ with $\operatorname{vcd}\left(\Gamma_{1} \times \Gamma_{2}\right)<v c d \Gamma_{1}+v c d \Gamma_{2}$. Nevertheless the logarithmic law holds if $\Gamma_{1}=\Gamma_{2}$. So this behavior of $v c d$ is analogous to that of the covering dimension dim for ANR-compacta [3]. The third application of the formula is the reduction of the following problem to a question of the existence of polyhedra with certain combinatorial properties.

Problem. Does there exist a Coxeter group $\Gamma_{n}$ with $n$-dimensional boundary and with $\operatorname{dim}_{\mathbb{Q}} \partial \Gamma_{n}=1$ ?

Note that groups with 2-dimensional and rationally 1-dimensional boundaries were constructed before $([4],[1],[2])$.

Received by the editors January 28, 1995.

1991 Mathematics Subject Classification. Primary 20F32, 55M10, 57S30; Secondary 16A60, 20J05, 51K10, 54E45.

The author was partially supported by NSF grant DMS-9500875.

(C) 1997 American Mathematical Society 
The problem for compacta without any relation to groups was solved for $n=2$ by Pontryagin in the early 30's, the corresponding compactum $X_{n}$ for $n=3$ was constructed by Kuzminov [5] only in the 60's, and in the 80's it was done for all $n$ [3]. For boundaries of Coxeter groups the problem is still unsolved for $n \geq 3$. In this paper we construct a finite 3 -dimensional simplicial complex with a given link. It gives an approach to the problem in the dimension three. According to our formula, to solve the problem it suffices to construct a rationally acyclic 3-dimensional simplicial complex with rationally acyclic links having nontrivial integral 3-cohomology.

Let $V$ be a finite set and let $I \subset V \times V$ be a symmetric subset. The group $\Gamma$ given by the presentation

$$
\left\langle V \mid v^{2}=1,(v w)^{m(v, w)}=1 \forall v \in V \forall(v, w) \in I ; 0<m(v, w)=m(w, v) \in \mathbb{Z}\right\rangle
$$

is called the Coxeter group. If $m(v, w)=2$ for all $(v, w) \in I$, then the group is called a right-angled Coxeter group. The group $\Gamma$ together with its presentation is called a Coxeter system $(\Gamma, V)$. Let $\mathcal{F}$ be the set of all nonempty subsets of $V$ that generate a finite subgroup of $\Gamma$. Then $\mathcal{F}$ is a partially ordered set (poset) by inclusion. Then [6] $\mathcal{F}$ is isomorphic to the poset of simplices of some simplicial complex $K=K(\Gamma, V)$. In the case of a right-angled Coxeter group we can construct $K(\Gamma, V)$ by the following rule. The vertices of $K$ are $V$. Two vertices $v$ and $w$ are joined by an edge if $(v, w) \in I$. A set of $n+1$ vertices spans an $n$-dimensional simplex iff all vertices are pairwise joined by edges [6]. The last property of a simplicial complex gives a definition of a flag complex [6].

\section{The LOCAL COHOMOlOGiCAL Dimension OF Simplicial COMPLEX AND THE FORMULA FOR $v c d_{F} \Gamma$}

Let $K$ be a simplicial complex. We recall that the collection of all simplices in $K$ containing a given vertex $v$ forms a complex $S t(v, K)$, called the star of $v$ in $K$, and the collection of all simplices in $S t(v, K)$ not containing $v$ forms a complex $L k(v, K)$, called the link of $v$ in $K$. We denote by $\beta^{1} K$ the first barycentric subdivision of $K$. For every simplex $\sigma \subset K$ we define the normal star $n s t(\sigma, K)$ of $\sigma$ in $K$ as a subcomplex of the star $\operatorname{St}\left(v(\sigma), \beta^{1} K\right)$ of the barycenter $v(\sigma)$ of the simplex $\sigma$ in $\beta^{1} K$ consisting of those simplices $\delta$ whose intersection with $\beta^{1} \sigma$ consists of $v(\sigma)$. The normal link $n l k(\sigma, K)$ of $\sigma$ in $K$ is the boundary of $n s t(\sigma, K)$, i.e. the subcomplex of $n s t(\sigma, K)$ consisting of all simplices having empty intersection with $v(\sigma)$.

We are going to use the same symbol for a simplicial complex and for its geometric realization.

Definition. The local cohomological dimension of a simplicial complex $K$ with respect to a coefficient group $G$ is

$$
l c d_{G} K=\max _{\sigma \in K}\left\{m \mid H^{m}(n s t(\sigma, K), n l k(\sigma, K) ; G) \neq 0\right\} .
$$

The global cohomological dimension [7] of a simplicial complex $K$ over $G$ is $c d_{G} K=\max \left\{m \mid H^{m}(K ; G) \neq 0\right\}$. Note that $H^{m}((n s t(\sigma, K), n l k(\sigma, K)) ; G)=$ $H^{m-1}(n l k(\sigma, K) ; G)$ and hence $l c d_{G} K=\max _{\sigma \in K}\left\{m \mid H^{m-1}(n l k(\sigma, K) ; G) \neq 0\right\}$.

These dimensions can differ from each other. For example, if $K$ is a triangulation of the join $\mathbb{R} P^{2} * \mathbb{R} P^{2}$ of the projective plane with itself, generated by some triangulation on $\mathbb{R} P^{2}$, then its dimensions over the integers and the rationals are $c d_{\mathbb{Z}} K=l c d_{\mathbb{Z}} K=5$ and $l c d_{\mathbb{Q}} K=4$ and $c d_{\mathbb{Q}} K=0$. 
Theorem 1. For every abelian group $G$ and every finite-dimensional simplicial complex $K$ there is the inequality $l c d_{G} K \geq c d_{G} K$.

We denote by $C K$ the cone over a simplicial complex $K$ with the natural triangulation.

Theorem 2. For every Coxeter group $\Gamma$ defined by a Coxeter system $(\Gamma, V)$ there is the equality $v c d_{F} \Gamma=l c d_{F} C K$ where $K=K(\Gamma, V)$ and $F$ is a principal ideal domain.

Every $r$-dimensional complex $K$ has a filtration $L_{0} \subset L_{1} \subset \ldots \subset L_{r}=K$ where $L_{i}=\bigcup_{d i m \sigma=r-i} n s t(\sigma, K)$. By induction we define the filtration $L_{0}^{\prime} \subset L_{1}^{\prime} \subset \ldots \subset$ $L_{r}^{\prime}=B(K, F)$ for a given abelian group $F$. We define polyhedra $L_{i}^{\prime}$ together with projections $p_{i}: L_{i}^{\prime} \rightarrow L_{i}$ such that $p_{i}$ is an extension of $p_{i-1}$ for every $i$. Define $L_{0}^{\prime}=L_{0}$ and $p_{0}=i d$. Assume that $p_{i-1}: L_{i-1}^{\prime} \rightarrow L_{i-1}$ is defined. For every $(r-i)$-simplex $\sigma$ we consider $A(\sigma)=p_{i-1}^{-1}(n l k(\sigma, K))$. Let $C(\sigma)$ be an $F$-acyclic polyhedron containing $A(\sigma)$ and of the least possible dimension. We attach $C(\sigma)$ to $L_{i-1}^{\prime}$ along $A(\sigma)$ and extend the map $p_{i-1}$ over $C(\sigma)$ such that the image of $C(\sigma)-A(\sigma)$ lies in $n s t(\sigma, K)-n l k(\sigma, K)$. Do that for all $\sigma$ to obtain $p_{i}: L_{i}^{\prime} \rightarrow L_{i}$. The resulting space $L_{r}$ is Bestvina's complex $B(K, F)$ of a complex $K$ with respect to a group $F$.

Note that the map $p=p_{r}: B(K, F) \rightarrow K$ has the property that the preimage of every normal star $n s t(\sigma, K)$ is $F$-acyclic.

Theorem 3 ([1]). For every Coxeter group $\Gamma$ defined by a Coxeter system $(\Gamma, V)$ the following equality holds:

$$
\operatorname{vcd}_{F} \Gamma=\operatorname{dim} B(C K(\Gamma, V) ; F)
$$

if $F$ is a principal ideal domain.

We define by induction the notion of a star-subcomplex $N$ of $K$. The empty set is a star-subcomplex. A union of some normal stars of $(r-i)$-dimensional simplices $n s t(\sigma, K)$ form a star-subcomplex of $K$ if all pairwise intersections of those normal stars are star-subcomplexes.

Proposition 1. For every star subcomplex $N \subset K, H^{n}(N ; F)=H^{n}\left(p^{-1}(N) ; F\right)$ for all $n$.

Proof. Induction, the Mayer-Vietoris sequence and the fact that

$$
\bar{H}^{*}\left(p^{-1}(n s t(\sigma, K)) ; F\right)=0
$$

imply $H^{n}(N ; F)=H^{n}\left(p^{-1}(N) ; F\right)$ for all $n$ and for every star-subcomplex $N \subset K$. This argument is well-known and is sometimes called the combinatorial VietorisBegle theorem.

Proposition 2. $\operatorname{dim} B(K, F)=l c d_{F} K$.

Proof. Let $l c d_{F} K=n$. Hence $H^{n-1}(n l k(\sigma, K) ; F) \neq 0$ for some simplex $\sigma$ in $K$. By Proposition 1 we have $H^{n-1}\left(p^{-1}(n l k(\sigma, K)) ; F\right) \neq 0$. Therefore, $H^{n-1}(A(\sigma) ; F) \neq$ 0 . Then by the construction $C(\sigma)$ is at least $n$-dimensional. Hence $\operatorname{dim} B(K, F) \geq$ $n$.

Now let $\operatorname{dim} B(K, F)=n$. Then by the construction of $B(K, F)$ there exists a simplex $\sigma \subset K$ with $H^{n-1}(A(\sigma) ; F) \neq 0$. Then by Proposition $1, H^{n-1}(n l k(\sigma, K) ; F)$ $\neq 0$ and hence $l c d_{F} K \geq n$. 
Now the proof of Theorem 2 follows by Proposition 2 and Theorem 3 .

Proof of Theorem 1. Assume that $c d_{F} K=n$. Consider Bestvina's complex $p$ : $B(K, F) \rightarrow K$. Proposition 1 implies that $H^{n}(B(K, F) ; F) \neq 0$ and, hence, $\operatorname{dim} B(K, F) \geq n$. Proposition 2 implies the proof.

Proposition 3. Let $C K$ be a cone over $K$. Then

$$
l c d_{F} C K=\max \left\{l c d_{F} K, c d_{F} K+1\right\} .
$$

Proof. For every simplex $\sigma \subset K \subset C K$ the normal link $n l k(\sigma, C K)$ is homeomorphic to the cone over the normal link $n l k(\sigma, K)$ and, hence, is cohomologically trivial. The normal link of the cone vertex $v$ is $K$. The normal link of any other simplex $\sigma$ containing $v$ is homeomorphic to $n l k(\delta, K)$ for some simplex $\delta$. If $c d_{F} K<l c d_{F} K$, then $l c d_{F} C K=l c d_{F} K$ by the above and hence the formula is true. If $i=c d_{F} K \geq l c d_{F} K$, then $l c d_{F} C K=i+1$ and the formula is true again.

\section{Applications of the Formula FOR $v c d_{F} \Gamma$}

Theorem 4. A Coxeter group $\Gamma$ has the following properties:

a) $v c d_{\mathbb{Q}} \Gamma \leq v c d_{G} \Gamma$ for any abelian group $G$,

b) $v c d_{\mathbb{Z}_{p}} \Gamma=v c d_{\mathbb{Q}} \Gamma$ for almost all primes $p$,

c) there exists a prime $p$ such that $v c d_{\mathbb{Z}_{p}} \Gamma=v c d \Gamma$.

Proof. a) Let $v c d_{\mathbb{Q}} \Gamma=n$. By Theorem 2 there exists $\sigma \subset C K$ such that

$$
H^{n-1}(n l k(\sigma, C K) ; \mathbb{Q}) \neq 0 .
$$

The Universal Coefficient Formula implies that the group $H^{n-1}(n l k(\sigma, C K) ; \mathbb{Z})$ has a nontrivial rank. Hence, $H^{n-1}(n l k(\sigma, C K) ; G) \neq 0$ and therefore, $l_{c} d_{G} C K \geq n$. Theorem 2 implies that $v c d_{G} \Gamma \geq n$.

b) Let again $v c d_{\mathbb{Q}} \Gamma=n$. Since we have only finitely many simplices $\delta \subset C K$ and finitely many $m \leq \operatorname{dim} C K$, only finitely many prime numbers are involved in the torsions of $H^{m}(n l k(\delta, C K) ; \mathbb{Z})$. Hence for almost all prime $p, H^{m}\left(n l k(\delta, C K) ; \mathbb{Z}_{p}\right)=$ 0 for $m \geq n$. Therefore, $l c d_{\mathbb{Z}_{p}} C K \leq n$. This together with a) proves $\mathrm{b}$ ).

c) Let $v c d \Gamma=r$; then by Theorem 2 there is a simplex $\sigma^{\prime} \subset C K$ such that $H^{r-1}\left(n l k\left(\sigma^{\prime}, C K\right) ; \mathbb{Z}\right) \neq 0$. Hence by virtue of the Universal Coefficient Formula, $H^{r-1}\left(n l k\left(\sigma^{\prime}, C K\right) ; \mathbb{Z}_{p}\right) \neq 0$. Hence, $l c d_{\mathbb{Z}_{p}} C K \geq r$ and hence, $l c d_{\mathbb{Z}_{p}} C K=r$. Theorem 2 implies that $v c d_{\mathbb{Z}_{p}} \Gamma=v c d \Gamma$.

Corollary 1. The equality $v c d(\Gamma \times \Gamma)=2 v c d \Gamma$ holds for every Coxeter group.

Corollary 2. A Boltyanski's compactum cannot be the boundary of a Coxeter group.

Proofs. Since the formula $v c d_{F} \Gamma \times \Gamma^{\prime}=v c d_{F} \Gamma+v c d_{F} \Gamma^{\prime}$ holds for every field $F$ [10], Theorem $4 \mathrm{c}$ ) implies the proof of Corollary 1. The characterizing property of Boltyanski's compactum $B$ is $\operatorname{dim} B \times B=3$ [5]. This property implies that $\operatorname{dim} B=\operatorname{dim}_{\mathbb{Z}} B=2$ and $\operatorname{dim}_{\mathbb{Z}_{p}} B=1$ for every prime $p$. Then in view of Theorem $4 \mathrm{c}$ ) and the following theorem Corollary 2 follows.

Theorem 5 (Bestvina-Mess [4]). Let $\partial \Gamma$ be the boundary of a Coxeter group $\Gamma$. Then for every abelian group $G, \operatorname{dim}_{G} \partial \Gamma=v c d_{G} \Gamma-1$. 
The other way to derive Corollary 2 from the Bestvina-Mess theorem consists of proving the following boundary formula $\partial\left(\Gamma \times \Gamma^{\prime}\right)=\partial \Gamma * \partial \Gamma^{\prime}$.

We recall that a compactum $X$ has the cohomological dimension $\operatorname{dim}_{G} X \leq n$ if for every closed subset $A \subset X$ the relative Cech cohomology group $\check{H}^{n+1}(X, A ; G)$ is trivial $([3],[5])$. Note that for finite dimensional compacta $\operatorname{dim} X=\operatorname{dim}_{\mathbb{Z}} X[5]$.

Corollary 3 ([2]). For every prime $p$ there is a Pontryagin surface $\Pi_{p}$ which is the boundary of some right-angled Coxeter group.

Proof. The Pontryagin surface $\Pi_{p}$ is defined by the properties: $\operatorname{dim}_{\mathbb{Q}} \Pi_{p}=\operatorname{dim}_{\mathbb{Z}_{q}} \Pi_{p}$ $=1$ for $q$ relatively prime with $p$ and $\operatorname{dim} \Pi_{p}=2$. Hence $\operatorname{dim}\left(\Pi_{p} \times \Pi_{q}\right)=3$ if $p$ and $q$ are relatively prime. If we take the Moore complex $M\left(\mathbb{Z}_{p}, 1\right)$ with an arbitrary triangulation $K$, then by Theorem $2 v c d_{F} \Gamma=l c d_{F} C K$ for the Coxeter group $\Gamma$ generated by $K$. By Proposition $3 l c d_{F} C K=\max \left\{l c d_{F} K, c d_{F} K+1\right\}$. It is easy to see that $l c d_{F} K=2$ for all $F$. Note that $c d_{F} K=2$ if $F=\mathbb{Z}_{p}$ and $c d_{F} K=1$ for $F=\mathbb{Q}, \mathbb{Z}_{q}$. Hence $v c d_{F} \Gamma=2$ if $F=\mathbb{Z}_{q}$ or $\mathbb{Q}$ and $v c d \Gamma=3$. Theorem 5 implies that the boundary $\partial \Gamma$ is a Pontryagin surface $\Pi_{p}$.

We recall that the boundary of the Coxeter system $(\Gamma, K)$ is the visual sphere at infinity of $\mathrm{CAT}(0)$ cubical complex $\Sigma$ associated with $(\Gamma, K)[6]$.

\section{Complexes With a Given LinK}

In this section we prove the following:

Lemma 1. For every 2-dimensional compact polyhedron $K$ there exists a finite simplicial complex $L$ such that the link $L k(v, L)$ of every vertex is homeomorphic to $K$.

In view of Theorem 2 and Proposition 3, Lemma 1, applied to $K$ equal to the real projective plane, almost gives a solution of the Problem for $n=3$ (see the Introduction). It gives a flag complex $L$ with $c d L=3$ and $l c d_{\mathbb{Q}} L=2$. If additionally we could require the equality $c d_{\mathbb{Q}} L=1$, then the Coxeter group $\Gamma$ defined by the simplicial complex $L$ should have dimensions $v c d \Gamma=4$ and $v c d_{\mathbb{Q}} \Gamma=2$. Hence the boundary $\partial \Gamma$ would be 3 -dimensional with $\operatorname{dim}_{\mathbb{Q}} \partial \Gamma=1$. Thus, to get a solution of the Problem one needs a complex $L$ of Lemma 1 with trivial rational 2-dimensional cohomology groups.

By the definition a cubical complex $\Sigma$ is the union of cubes $C$ with intersections along faces. A locally finite cubical complex has the following natural metric on it. All cubes are considered isomorphic to the standard euclidean cube of length one and the distance between two points in different cubes is defined as the infimum of the length of curves joining these two points.

The following theorem can be extracted from [6].

Theorem 6. For every right-angled Coxeter system $(\Gamma, S)$ there exists a cubical complex $\Sigma=\Sigma(\Gamma, S)$ with the following properties:

1) $\Gamma$ acts by isometries on $\Sigma$ with finite isotropy groups and with the orbit space homeomorphic to the cone $C K$ over $K=K(\Gamma, S)$.

2) There is the natural imbedding $j$ of $C K$ in $\Sigma$ such that the projection onto orbit space is a retraction and hence $\Gamma\left(D_{0}\right)=\Sigma$, where $D_{0}=j(C K)$.

3) Let $v_{0}$ be the cone point of $D_{0}$; then $D_{0}$ is the image of 1/2-homothety centered at $v_{0}$ of the cubical star $S t\left(v_{0}, \Sigma\right)=$ the union of all cubes in $\Sigma$ having $v_{0}$ as a vertex. 
4) There is a bijection $s \leftrightarrow\left\{v_{0}, s\left(v_{0}\right)\right\}$ between $S$ and the set of edges of $\Sigma$ emanating from $v_{0}$ such that $s$ as an element of $\Gamma$ takes $v_{0}$ to $s\left(v_{0}\right)$. Edges $\left[v_{0}, s_{1}\left(v_{0}\right)\right], \ldots,\left[v_{0}, s_{k}\left(v_{0}\right)\right]$ form a $k$-cube in the complex $\Sigma$ if and only if $\left(s_{i}, s_{j}\right) \in I$ for all $i, j \leq k, i \neq j$.

5) $\Gamma$ acts freely and transitively on the set of all vertices of $\Sigma$.

6) $\Sigma$ is a $C A T(0)$ geodesic space and, hence, is contractible.

The following theorem is due to Tits and Selberg.

Theorem 7 ([6]). Every Coxeter group $\Gamma$ has a torsion free subgroup $\Gamma^{\prime}$ of finite index.

Note that in the case of right-angled Coxeter groups the commutator group $[\Gamma, \Gamma]$ can be taken as $\Gamma^{\prime}$.

Proof of Lemma 1. Consider a flag triangulation on $K$ with the set of vertices $S$ and consider the corresponding Coxeter system $(\Gamma, S)$. Show that the cubical complex $\Sigma=\Sigma(\Gamma, S)$ of Theorem 6 admits a triangulation with the same set of vertices. In the domain $2 D_{0}=\operatorname{St}\left(v_{0}, \Sigma\right)$ for every 2-dimensional cube $F$ containing $v_{0}$ we consider a triangulation of $F$ generated by the diagonal $\left[v_{0}, w\right]$. For every 3 -dimensional cube $C$ in $2 D_{0}$ consider a triangulation generated by diagonals $\left[v_{0}, w\right]$ in 2 -faces $F$ of $C\left(v_{0} \in F\right)$ and antiparallel diagonals in opposite 2-faces $\bar{F}\left(F \cap\left\{v_{0}\right\}=\emptyset\right)$. If $C$ and $C^{\prime}$ are two cubes in $2 D_{0}$ with common face $F$, then $v_{0} \in F$ and hence the triangulations agree on these two cubes. Thus we have a triangulation on $2 D_{0}$ with the same set of vertices as the cubical complex $2 D_{0}$.

Denote by $G$ a subgroup of $\Gamma$ consisting of all elements having an even length in the alphabet $S$. Note that $G$ is well-defined. Indeed, since all the relations in the presentation of the group $\Gamma$ have an even length, the equality $s_{1} s_{2} \ldots s_{n}=1$ is impossible for an odd $n$ by Tits' theorem [8]. Note that $G\left(2 D_{0}\right)=\Sigma$. Indeed, for every $g \in \Gamma$ and for every cube $C \subset 2 D_{0}$, we have

$$
g(1 / 2 C) \subset \bigcup_{s \in S} g s(C) \subset G\left(2 D_{0}\right) \quad \text { and } \quad \Sigma=\bigcup_{C \subset 2 D_{0}} \bigcup_{g \in \Gamma} g(1 / 2 C) .
$$

By Theorem 62$)$, we have $\Sigma=\Gamma\left(D_{0}\right)=\Gamma(\bigcup 1 / 2 C) \subset G\left(2 D_{0}\right)$. We define a triangulation on $\Sigma$ by translations of that on $2 D_{0}$ by the group $G$. To show that this definition is correct it suffices to show that for every 2-dimensional cube $\Phi$ in $\Sigma$ and for any two 2-cubes $F$ and $F^{\prime}$ in $2 D_{0}$ with $g(F)=\Phi=g^{\prime}\left(F^{\prime}\right), g, g^{\prime} \in G$, the images of the diagonals participating in the triangulation on $2 D_{0}$ are the same. Consider $g^{-1} g^{\prime}\left(F^{\prime}\right)=F$. Without loss of generality we may assume that $v_{0} \in F \cap F^{\prime}$. Since $g^{-1} g^{\prime} \in G$, by Theorem 64$), g^{-1} g^{\prime}\left(v_{0}\right)$ cannot be an adjoined vertex with $v_{0}$. Hence $g^{-1} g^{\prime}\left(v_{0}\right)=v_{0}$ or the other end of the diagonal. In both cases $g^{-1} g^{\prime}$ of the diagonal $\left[v_{0}, w^{\prime}\right]$ of $F^{\prime}$ is the diagonal $\left[v_{0}, w\right]$.

We may assume that a torsion free group $\Gamma^{\prime}$ of Theorem 7 is a subgroup of $G$. For every $g \in \Gamma^{\prime}$ and for every cube $C \subset D_{0}$, we have $g(C) \cap C=\emptyset$. Indeed, if vertices $v$ and $g(v)$ belong to $C$, then there exists an element $q \in \Gamma$ of the form $s_{i}$ or $s_{i} s_{j}$ or $s_{i} s_{j} s_{k}$ with $q(v)=g(v)$. By 4$)$ of Theorem $6 q$ is of a finite order. By 5$)$ of Theorem $6 q=g$. It contradicts the fact that $\Gamma^{\prime}$ is torsion free. Therefore $\Gamma^{\prime}$ acts freely on $\Sigma$ preserving the triangulation. For every simplex $\sigma$ of the triangulation all vertices lie in different orbits of $\Gamma^{\prime}$. Hence the orbit space $L=\Sigma / \Gamma^{\prime}$ is a simplicial complex. By 2),3),5) of Theorem 6 the link of every vertex $v \in \Sigma$ is homeomorphic to $K$, therefore $L k(w, L)$ is homeomorphic to $K$ for all vertices $v \in L$. 
We note that the main result of this paper (Theorem 2) can be derived also from recent computations of the cohomology of Coxeter groups by M. Davis [11].

The author is thankful to the referee for valuable remarks.

\section{REFERENCES}

1. M. Bestvina, The virual cohomological dimension of Coxeter groups, Geometric Group Theory Vol 1, LMS Lecture Notes 181, 19-23. MR 94g:20056

2. A.N. Dranishnikov, Boundaries and cohomological dimensions of Coxeter groups, Preprint (1994).

3. A. Dranishnikov, Homological dimension theory, Russian Math. Surveys 43 (1988), 11-63. MR 90e:55003

4. M. Bestvina, G. Mess, The boundary of negatively curved groups, Jorn. of Amer. Math. Soc. 4 (no. 3) (1991), 469-481. MR 93j:20076

5. V.I. Kuzminov, Homological dimension theory, Russian Math. Surveys 23 (5) (1968), 1-45. MR 39:2158

6. M. Davis, Nonpositive curvature and reflection groups, Preprint (1994).

7. K.S. Brown and P.J. Kahn, Homotopy dimension and simple cohomological dimension of spaces, Comment. Math. Helv. 52 (1977), 11-127. MR 55:11251

8. K.S. Brown, Buildings, Springer-Verlag, New York, Berlin, 1989. MR 90e:20001

9. K.S. Brown, Cohomology of groups, Springer-Verlag, New York, Heidelberg, Berlin, 1982. MR 83k:20002

10. R. Bieri, Homological dimension of discrete groups, Queen Mary College, London, 1976. MR 57:6224

11. M. Davis, The cohomology of a Coxeter group with group ring coefficients, Preprint (1995).

Department of Mathematics, University of Florida, Gainesville, Florida 32611

E-mail address: dranish@math.ufl.edu 